\title{
Mathematical modelling of the chronoamperometric response of an array of rectangular microelectrodes
}

\author{
Spas D Kolev ${ }^{1}$, Jo H M Simons and Willem E van der Linden \\ Laboratory of Chemical Analysss, Department of Chemical Technology, University of Twente, PO Box 217, \\ 7500 AE Enschede (Netheriands)
}

(Recerved 13th August 1992)

\begin{abstract}
A general mathematical model describing the response of an array of flat amperometric electrodes with arbitrary size and spatial distribution at the bottom of a measurng cell with rectangular walls and finte dimensions is outlined It is based on the three-dimensional diffusion equation with initial and boundary conditions corresponding to the physical situation which was numencally solved by the implicit alternating-direction finte-difference method The accuracy of the numencal solution was confirmed by theoretical and experimental results obtaned by other authors By comparing the chronoamperometric curves of the individual electrodes and by examining the spatial concentration distribution in the measuring cell conclusions can be drawn concerning the mutual influence of the individual electrodes for a given geometry of the array and the dimensions of the measuring cell This will allow the designing of arrays and selectung the proper measuring cell dimensions resultıng in minmal sensor interferences Chronoamperometric curves show the time required for attaining quasi steady state and the corresponding current value Illustrative examples are presented
\end{abstract}

Keywords Amperometry, Chronoamperometry, Mathematical modelling, Microelectrode arrays

In static solutions microelectrodes exhibit a number of advantages related to their size $(\mathrm{e} g$, in vivo measurements [1]) and electrochemical properties [2-24] in comparison with conventional macroelectrodes The most important electrochemical properties are the following (i) enhanced current densities due to non-linear diffusion [2-13] which results in a rapid establishment of the quasi steady-state in chronoamperometry [6-8,11,12], sigmoidal cyclic voltammograms with reversible couples for moderate scan rates $[2,14-$ 17], and increased sensitivity to small deviations from reversibility, allowing the measurement of

Correspondence to W E van der Linden, Laboratory of Chemical Analysis, Department of Chemical Technology, University of Twente, PO Box 217, NL-7500 AE Enschede (Netherlands)

1 Permanent address Faculty of Chemustry, University of Sofia, 1 James Bouchuer Ave, BG-1126 Sofia (Bulgana) high rate constants (e g , up to $400 \mathrm{~cm} \mathrm{~s}^{-1}$ [18]), (11) low ohmic potential drop which allows measurements to be performed in highly resistive media [19-24], (11) reduced double-layer capac1tance due to the small surface area which, together with the low ohmic drop, allows measurement of faradaic currents at very short times and the extension of cyclic voltammetry to high scan rates [21]

The currents measured by microelectrodes, despite the non-linear diffusion effects, remain substantially lower in absolute values ( $\mathrm{g}$, down to the order of femtoamperes) than those of conventional macroelectrodes This drawback, though not crucial in view of the modern instrumentation avallable nowadays, can easily be overcome by using ensembles of microelectrodes connected in parallel [25-29] Depending on the method of manufacturing, (ultra)microelectrode arrays can 
have regular (e g, microlithography [27]) or irregular (e g , composite electrodes [29]) geometry In addition to the valuable properties of single microelectrodes mentioned above the arrays of $\mathrm{ml}$ croelectrodes offer some additional advantages worth mentioning It has been established both theoretically and experimentally that an array of microelectrodes in the long-tıme range $(1 \mathrm{e}$, when the non-linear diffusion fields of the individual microelectrodes overlap) behaves like a macroelectrode (Cottrellian diffusion) with an area equal to the total geometric area of the array, not only to the sum of the areas of the individual microelectrodes comprising the electroactive area of the array [30-34,36-38] This effect results on one hand in a considerable improvement in the signal-to-noise ratio of the array because despite the fact that the faradaic signal is proportional to the total geometric area (electroactive and nonelectroactive area), the noise remains proportional only to the electroactive area of the array $[34,39,40]$ On the other hand it allows a considerable economy of the electroactive matenal which very often is a nobel metal [26-28] The possibility to address individually each microelectrode in an array can be used for simultaneous multicomponent analysis or for study of reaction mechanism by simultaneously detecting the participating species in the reaction The effectiveness of this approach can be further enhanced by modifying the individual microelectrodes in order to improve the selectivity

For better understanding of the processes occurring at ensembles of microelectrodes and for their optimal design an adequate mathematical model is required Models based on analytical $[30,32,37]$ and numerical $[31,33-36]$ solution of the partial differential equation expressing Ficklan diffusion to arrays of disk, ring or square microelectrodes under vanous simplifying assumptions have been proposed before Lindemann and Landsberg [30] assumed uniform distr1bution of microdisk electrodes in a rigid hexagonal array They reduced the diffusion problem for such an ensemble to the diffusion to an array of non-interactıng semi-infinite contiguous cylindrical unit cells with a concentrically situated circular active site at their bases The equation derived for the diffusion current was based on the Cottrell equation [40] in which the term for the diffusion layer was corrected according to the results of Smythe [41] However, those results are valid for steady-state conditions only and their application to the transient problem of chronopotentiometry resulted in discrepancies between theory and experument [30] Levart et al [31] treated the diffusion to an array of periodically distributed square active sites under steady-state conditions and the results obtained are similar to those of Lindemann and Landsberg [30] Gueshı et al [32] developed a model using a representation of the microelectrode array similar to that of Lindemann and Landsberg [30] and assumed a steady-state radial diffusion The resulting system of differential equations with their initial and boundary conditions corresponds exactly to that for an electron transfer preceded by a first-order chemical reaction The analytical solution of the model gives accurate results for the current at short (1 e , semı-infinite linear diffusion to the electroactive area) and long (1 e, semi-infinite linear diffusion to the total geometric area) times For intermediate times ( $1 \mathrm{e}$, non-linear diffusion to the individual microelectrodes) the predicted current was found to be too low, the deviation increasing with decreasing the fraction of the electroactive area Reller et al [33] numerically solved the model proposed by Gueshi et al [32] taking into consideration the transient character of radial diffusion by an explicit finite-difference technique Good agreement between reported experumental data [30] and results based on the simulation for the whole time range was observed Weisshaar and Tallman [34] derived a model for carbon-based composite electrodes assuming that they consist of two ensembles of microelectrodes with different geometrical dimensions behaving independently of one another so that the total electrode current was simply a weighted summation of the two contributions, each described by the equation of Gueshı et al [32] Shoup and Szabo [35] numencally solved the problem treated in literature $[30,32,33]$ using the hopscotch algorithm They derived an empirical expression based on the equation for the current at an isolated microdisk electrode [6], which accu- 
rately reproduced the results of the simulations for all tumes and for all fractional coverages The authors found that for very short times $(t \rightarrow 0)$ the ensemble current should not coincide with the Cottrell results [30-33] but be displaced from them by $\pi / 4$ No experimental or simulation proof for this theoretical prediction can be found in the literature Cassidy et al [36] extended the problem treated in literature [30-34] to the case of a reversible simple electron-transfer reaction so that the concentrations of the reduced and the oxidized species at each electroactıve disk surface were coupled by the Nernst equation The orthogonal collocation method was used for the solution of the corresponding diffusion equations for the electroactive species The simulation results agreed farrly well with those of Reller et al [33] and those of Shoup and Szabo [35], and with the experimental data presented [30] Scharifker [37] developed a simple analytical approach for calculating the time-dependent diffusion current to square, hexagonal and random arrays of mlcrodisk electrodes using only the analytical expressions for the non-linear diffusion current to a single microdisk electrode [3] and the Cottrell equation [40] The approach is based on considering the overlap of equivalent diffusion zones defined by the author as the circular area incorporatıng a microdisk electrode to which linear diffusion will produce the same effect as the actual non-linear diffusion to the same electrode The overlap was calculated through the corresponding exact geometrical constructions in the case of square and hexagonal arrays or by applying the Avrami-Kolmgorov theorem in case of random arrays Despite the substantial simplifying assumptions introduced by the author the analytical expressions obtained are in fairly good agreement with the theoretical results of Shoup and Szabo [35] and the experumental data presented by Guesh1 et al [32]

All the models mentioned above possess several drawbacks limitıng their generality, the most important of them being the following

(1) Only ordered arrays with a high degree of symmetry (e g, square and hexagonal geometry) are considered which allows the more compl1cated diffusional problem to an array to be sim- plified to the case of diffusion to a single electrode The equation of Scharifker [37] for the diffusion current at randomly distributed overlapping microdisk electrode arrays has not been experimentally confirmed and due to the considerable simplifications made in its derivation it is difficult to predict its validity for real random arrays Modern microlithographic techniques allow the manufacturnng of arrays with various geometries and different degrees of symmetry which cannot be described mathematically by the existing models

(2) Only microdisk arrays have been considered except for reference [31] where the treatment of non-linear steady-state diffusion to an ordered array of square microelectrodes is reported

(3) The arrays are assumed to be infinitely wide and long with constant concentration of the electroactive species far from their surface These simplifying assumptions do not allow to take into account the effects of the walls of real electrochemical measuring cells on the response of the arrays in them, $1 \mathrm{e}$, the models are not suitable for the description of chronocoulometric measurements accompaned by depletion of the electroactive species in the whole volume of the measuring cell or to predict to what extent a real measuring cell can be miniaturized without affecting the chronoamperometric response Together with point 1 , this assumption excludes any interference in the responses of the individual microelectrodes, $1 \mathrm{e}$, the electrodes should exhibit identical responses For this reason shielding effects of electrodes, where because of the geometry of the array the access of the electroactive species is restricted, cannot be taken into account

In the present paper the development of a model overcoming the drawbacks of the models existing in the literature mentioned above is reported

DEVELOPMENT OF THE MATHEMATICAL MODEL

The mathematical model proposed in the present study is based on the following assumptions 


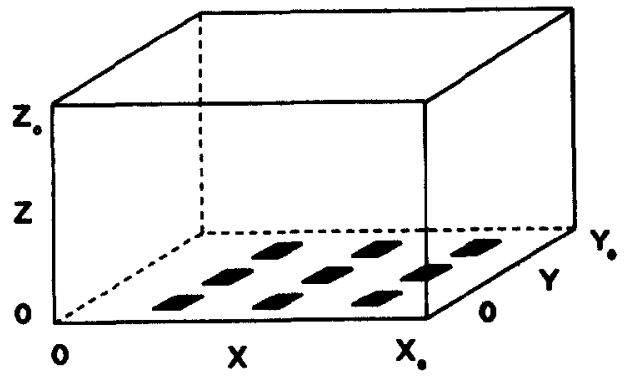

Fig 1 Scheme of the measuring cell with an array of 9 rectangular electrodes at its bottom

(1) the mass transfer is the result only of Fickian diffusion, $1 \mathrm{e}$, no migration effects are considered, (11) the walls of the measuring cell, which are assumed to be rectangles, are impermeable to the electroactive species inside it (Fig 1), (111) the depletion of the chemical species is a result only of the heterogeneous electrochemical reaction taking place at the electrodes which are situated at the bottom of the measuring cell (Fig 1), (Iv) a simple reversible charge-transfer reaction is considered and the potentials applied at all microelectrodes, which are not necessarily the same, are assumed to deviate from the formal potential $E_{0}$ to such an extent that exther the anodic or the cathodic reaction predominates, (v) the microelectrodes were assumed to be rectangular in shape and to lie at the same level as the bottom of the measuring cell

The mathematical model consists of the $\mathrm{d}_{1}$ mensionless Fick's second law (Eqn 1) The symbols and their definitions are given in Table 1

$\frac{\partial C}{\partial \theta}=\frac{\partial^{2} C}{\partial^{2} X}+\frac{\partial^{2} C}{\partial^{2} Y}+\frac{\partial^{2} C}{\partial^{2} Z}$

The initial conditions of Eqn 1 are

$$
\begin{gathered}
C(0, X, Y, Z)=1 \text { for } 0 \leq X \leq X_{0}, \\
0 \leq Y \leq Y_{0} \text { and } 0 \leq Z \leq Z_{0}
\end{gathered}
$$

The boundary conditions for the walls of the measuring cell excluding the electrodes are

$$
\left(\frac{\partial C}{\partial X}\right)_{X=0}=0 \text { for } 0 \leq Y \leq Y_{0} \text { and } 0 \leq Z \leq Z_{0}
$$

$$
\begin{aligned}
& \left(\frac{\partial C}{\partial X}\right)_{X=X_{0}}=0 \text { for } 0 \leq Y \leq Y_{0} \text { and } 0 \leq Z \leq Z_{0} \\
& \left(\frac{\partial C}{\partial Y}\right)_{Y=0}=0 \text { for } 0 \leq X \leq X_{0} \text { and } 0 \leq Z \leq Z_{0} \\
& \left(\frac{\partial C}{\partial Y}\right)_{Y=Y_{0}}=0 \text { for } 0 \leq X \leq X_{0} \text { and } 0 \leq Z \leq Z_{0}
\end{aligned}
$$

$\left(\frac{\partial C}{\partial Z}\right)_{Z=0}=0$ for $(X, Y) \notin S$

$\left(\frac{\partial C}{\partial Z}\right)_{Z=Z_{0}}=0$ for $0 \leq X \leq X_{0}$ and $0 \leq Y \leq Y_{0}$

where $S$ is the electroactive area of the array The boundary conditions for the electrodes in the case of slow or moderate rate of the heterogeneous charge transfer with respect to the masstransfer rates are given by Butler-Volmer equation

$\left(\frac{\partial C}{\partial \theta}\right)_{Z=0}=C_{Z=0} K_{0} \exp \left[\frac{\alpha n F}{R T}\left(E-E_{0}^{\prime}\right)\right]$

for $(X, Y) \in S$

while if the reaction is very fast the concentrations at the electrodes can be assumed as $0,1 \mathrm{e}$,

$C_{Z=0}=0$ for $(X, Y) \in S$

The dimensionless current $\left(I_{l}\right)$ monitored at each individual electrode and for the whole array $(I)$ can be calculated by

$I_{l}=\int_{s_{l}} \int\left(\frac{\partial C}{\partial Z}\right)_{Z=0} \mathrm{~d} X \mathrm{~d} Y / \int_{s_{t}} \int \mathrm{d} X \mathrm{~d} Y$

where $s_{t}$ is the area of the $t$ th microelectrode

$I=\sum_{i=1}^{i=N} I_{i}$

\section{NUMERICAL SOLUTION OF THE MODEL}

The implicit alternatıng-direction finite-difference method [42] has been successfully applied 
TABLE 1

Symbols and definitıons

a Coefficients defined in Table 3

$b \quad$ Coefficients defined in Table 3

c Concentration $\left(\mathrm{mol} \mathrm{m} \mathrm{m}^{-3}\right)$

$c_{0} \quad$ Initial concentration $\left(\mathrm{mol} \mathrm{m}^{-3}\right)$

$C=c / c_{0}$ Dimensionless concentration

$d \quad$ Step coefficient (Table 2)

$D \quad$ Diffusion coefficient $\left(\mathrm{m}^{2} \mathrm{~s}^{-1}\right)$

E Potential (V)

$E_{0}^{\prime} \quad$ Formal potential (V)

$F \quad=96486332$ Faraday constant $\left(\mathrm{C} \mathrm{mol}^{-1}\right)$

$l_{t} \quad$ Current at the $i$ th microelectrode (A)

$I_{t} \quad=l_{t} L / n F D s_{t} c_{0}$ Dimensionless current of the $t$ th electrode

I $=\sum I_{t}$ Dimensionless current of the array

$J_{t} \quad=I_{t} s_{t} / L$ Normalized current of the $t$ th microelectrode $(\mathrm{m})$

$J=\sum J_{1}$ Normalized current of the array (m)

$k_{0} \quad$ Standard heterogeneous rate constant $\left(\mathrm{s}^{-1}\right)$

$K_{0} \quad=L^{2} k_{0} / D$ Dimensionless standard heterogeneous rate constant

$L \quad$ Characteristic length (m)

$M \quad$ Total number of grid points in $X\left(M_{x}\right), Y\left(M_{y}\right)$, or $Z\left(M_{z}\right)$ direction of the spatial gnd (defined in Table 3)

$n \quad$ Number of electrons exchanged

$N \quad$ Number of grid points in $X\left(N_{x}\right), Y\left(N_{y}\right)$, or $Z\left(N_{z}\right)$ direction in a uniform or non-uniform region of the spatial grid

$R \quad=83145$ Gas constant $\left(\mathrm{J} \mathrm{K}^{-1} \mathrm{~mol}^{-1}\right)$

$s_{1} \quad$ Area of the $i$ th microelectrode $\left(\mathrm{m}^{2}\right)$

$S \quad$ Electroactive area of the array $\left(\mathrm{m}^{2}\right)$

$t$ Time (s)

$T \quad$ Absolute temperature (K)

$x, y, z \quad$ Directed distances in a cartesian coordinate system (m)

$X, Y, Z=x / L, y / L, z / L$ Dimensionless directed distances in a cartesian coordinate system ${ }^{a}$

Greek letters

$\alpha \quad$ Transfer coefficient in Butler-Volmer equation

$\Delta \theta \quad$ Dimensionless time increment

$\Delta \phi_{0} \quad$ Dimensionless spatial increment in the uniform space grid region

$\Delta X_{1} \quad$ Dimensionless spatial increment in $X$ direction (Table 2)

$\Delta Y_{1} \quad$ Dimensionless spatial increment in $Y$ direction (Table 2)

$\Delta Z_{1} \quad$ Dimensionless spatial increment in $Z$ direction (Table 2)

$\theta \quad=D t / L^{2}$ Dimensionless time

The subscripts of $X, Y$, and $Z$ are explained in Figs 1 and for the numerical solution of partial differential equations describing multidimensional mass transfer $[43,44]$ The method is unconditionally stable and the corresponding sets of implicit difference equations in the $X, Y$, and $Z$ directions have tridiagonal matrices and allow straightforward solution by a Gaussian elımination method [42] The characteristic length of an array $(L)$ was defined as the shorter length of the smallest rectangle in which all the individual microelectrodes of the array are confined For the solution of Eqn 1, which is a three-dimensional transient diffusion equation, a modification of the two-dimensional implicit alternating-direction method [42], proposed by Brian [45], was chosen In order to reduce the computation time, a mixed un1form/non-uniform space gnd was used. In the area where the electrodes were situated, $1 \mathrm{e}, X_{1}$ $\leq X \leq X_{2}, \quad Y_{1} \leq Y \leq Y_{2}, \quad Z \leq Z_{1} \quad\left(F_{1 g} \quad 2\right)$, an isotropic and in all three directions uniform space grid was used The spatial increment $\left(\Delta \phi_{0}\right)$ was selected in such a way that the edges of the electrodes coincide as much as possible with the grid lines Outside this area spatial increments along all the three coordinate axes increase with distance from the area where the electrodes are located (Fig 2) The size of the individual increments was determuned as elements of an arithmetic progression with a basic element equal to the spatial increment $\left(\Delta \phi_{0}\right)$ in the uniform-grid region and step coefficients, $d_{x}, d_{y}$, and $d_{z}$ chosen in such a way so that $X_{1}, Y_{1}$ and $Z_{0}-Z_{1}$ (Fig 2) are subdivided by an integer number of grid points, $N_{x}^{0}, N_{y}^{0}$, and $N_{z}^{1}$, respectively (Table 2) The finite-difference formulas for the firstand second-order derivatives necessary for constructing the implicit finite-difference equations were obtained from the Taylor expansion [42] The finite-difference formulas for the $X$ derivatives are given in Table 3 and they are similar to those in the $Y$ and $Z$ directions The non-unform space grid outlined above allows the description of the concentration field to be made in greater detail closer to the electrodes and in lesser detall at a greater distance from them where the variations in the concentration gradients are smaller and less grid points are necessary for their accurate determınation 

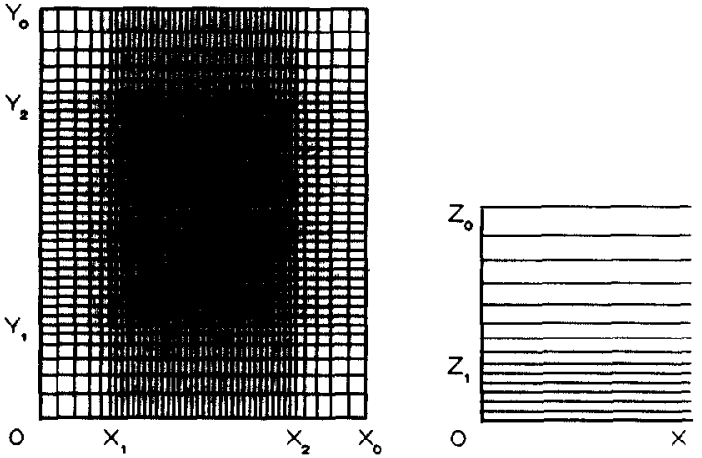

Fig 2 Spatial grid in the measuring cell Left $X Y$ plane at $Z=0$, nght $X Z$ plane at arbitrary $Y$

The double integral in Eqn 3 was calculated by the consecutive application of Simpson's rule [42]

An important step in the model simulation is the accurate calculation of the flux of the electroactive species towards the surface of the microelectrodes, $1 \mathrm{e},(\partial C / \partial Z)_{Z=0}$ at $(X, Y) \in S$ This task could pose severe difficulties because it is well known that numerical differentiation is an inherently less accurate process than numerical integration utilized for solving Eqn 1 Another reason to be cautious in this particular case is the fact that the concentration gradient in the $Z$ direction (Eqn 3 ) changes very rapidly with $Z$ in the neighbourhood of the microelectrodes Two different approaches for calculatıng $(\partial C / \partial Z)_{Z=0}$

\section{TABLE 3}

Finite-difference representation of $(\partial C / \partial X)$ and $\left(\partial^{2} C / \partial X^{2}\right)$ in the case of non-uniform space grid

(Subscripts $J$ and $k$ are omitted for simplicity)

\begin{tabular}{ll}
\hline$X$ & Finite-difference formulas \\
\hline $0 \leq X \leq X_{0}$ & $(\partial C / \partial X)_{X-X_{t}}=\left(C_{t}-C_{t-1}\right) / \Delta X_{t}$ \\
\hline$X=0^{\mathrm{a}}$ & $\left(\partial^{2} C / \partial X^{2}\right)_{X=0}=2\left(C_{1}-C_{0}\right) /\left(\Delta X_{1}\right)^{2}$ \\
$0<X<X_{0}$ & $\left(\partial^{2} C / \partial X^{2}\right)_{X=X_{1}}=\left(a_{t} C_{t-1}-2 C_{t}+b_{t} C_{t+1}\right) /$ \\
& $\left(\Delta X_{t} \Delta X_{t+1}\right)$ \\
$X=X_{0}$ & $\left.\partial^{2} C / \partial X^{2}\right)_{X=X_{0}}=2\left(C_{M_{x}-1}-C_{M_{x}}\right) /\left(\Delta X_{M_{x}-1}\right)^{2}$ \\
where & $a_{t}=2 \Delta X_{t+1} /\left(\Delta X_{t}+\Delta X_{t+1}\right)$ \\
& $b_{t}=2 \Delta X_{t} /\left(\Delta X_{t}+\Delta X_{t+1}\right)$ \\
& $M_{x}=N_{x}^{0}+N_{x}^{1}+N_{x}^{2}$
\end{tabular}

${ }^{a}$ Refers only to inactive area of the array where $(\partial C / \partial X)_{X=0}$ $=0$

at $(X, Y) \in S$ were investigated for finding the most appropriate one According to the first approach the function $C(X, Y, Z)=f(Z)$ was smoothed in the uniform space grid region ( $1 \mathrm{e}$, $\left.Z \leqslant Z_{1}, F_{1 g} 2\right)$ by the Savitzky-Golay algorithm [46] using a quadratic polynomal The derivative $(\partial C / \partial Z)_{Z-0}$ was obtained by subsequent analyt1cal differentiation of the least-square quadratic polynomial The second approach was based on approximating the function $C(X, Y, Z)=f(Z)$ with an interpolating polynomial of $n$th degree [42] To decide whether the flux should be calculated by a smoothing or interpolating polynomial and with what degree a comparison was made

TABLE 2

Calculation of the $X, Y$, and $Z$ increments in the mixed uniform/non-uniform spatial grid

\begin{tabular}{|c|c|c|c|}
\hline & Number of points & Region & Increment \\
\hline \multirow{3}{*}{${ }^{l}$} & $-N_{x}^{0}-1$ & $0-X_{1}$ & $\Delta X_{t+1}=\left[1+\left(N_{x}^{0}-\imath-1\right) d_{x}\right] \Delta \phi_{0}$ \\
\hline & $-N_{x}^{0}+N_{x}^{1}-1$ & $X_{1}-X_{2}$ & $\Delta X_{t+1}=\Delta \phi_{0}$ \\
\hline & $N_{x}^{0}+N_{x}^{1}-N_{x}^{0}+N_{x}^{1}+N_{x}^{2}-1$ & $X_{2}-X_{0}$ & $\Delta X_{b+1}=\left[1+\left(l-N_{x}^{0}-N_{x}^{1}\right) d_{x}\right] \Delta \phi_{0}$ \\
\hline \multirow{3}{*}{$\begin{array}{l}\text { J } \\
Y\end{array}$} & $0-N_{y}^{0}-1$ & $0-Y_{1}$ & $\Delta Y_{J+1}=\left[1+\left(N_{y}^{0}-J-1\right) d_{y}\right] \Delta \phi_{0}$ \\
\hline & $-N_{y}^{0}+N_{y}^{1}-1$ & $Y_{1}-Y_{2}$ & $\Delta Y_{j+1}=\Delta \phi_{0}$ \\
\hline & $N_{y}^{0}+N_{y}^{1}-N_{y}^{0}+N_{y}^{1}+N_{y}^{2}-1$ & $Y_{2}-Y_{0}$ & $\Delta Y_{j+1}=\left[1+\left(J-N_{y}^{0}-N_{y}^{1}\right) d_{y}\right] \Delta \phi_{0}$ \\
\hline $\begin{array}{l}k \\
Z\end{array}$ & $\begin{array}{ll}0 & -N_{z}^{0}-1 \\
N_{z}^{0} & -N_{z}^{0}+N_{z}^{1}-1\end{array}$ & $\begin{array}{l}0-Z_{1} \\
Z_{1}-Z_{0}\end{array}$ & $\begin{array}{l}\Delta Z_{k+1}=\Delta \phi_{0} \\
\Delta Z_{k+1}=\left[1+\left(k-N_{z}^{0}\right) d_{z}\right] \Delta \phi_{0}\end{array}$ \\
\hline
\end{tabular}


with an exasting analytical solution for the flux As such Cottrell's equation [40] was chosen

$I=(\pi \theta)^{-1 / 2}$

To meet the conditions under which Eqn 1 is valid, this equation was solved under the assumption that the entire bottom of the measuring cell was electroactive The smoothing of $C(X, Y, Z)$ $=f(Z)$ for $(X, Y) \in S$ was done using the concentration in the first 2-5 gnd points from the bottom of the measuring cell in the $Z$ direction The quadratic approxımatıng polynomial utilized for calculatung $(\partial C / \partial Z)_{Z=0}$ was of the order 1-5 In all cases very good agreement was observed for longer times while at short times the accuracy of the different approaches for calculating the flux differed The lowest values of the mean relative error and the square root of the mean squared error between the chronoamperometric curves calculated by Cottrell's equation (Eqn 5 ) and the numerical solution of Eqn 1 were obtained in the case of 4th order polynomial approximation

By varying the length of the spatial increment $\left(\Delta \phi_{0}\right)$ while keeping the time increment constant (1 e , $\Delta \theta=617 \times 10^{-5}$ corresponding to $\Delta t=005$ s) it was found that the numerical and analytical solutions are practically indistınguishable from each other for $\Delta \phi_{0} \leq 0012$ (Fig 3)

It should be taken into consideration that the values of the dimensionless currents $\left(I_{2}\right)$ of the individual microelectrodes in an array depend on

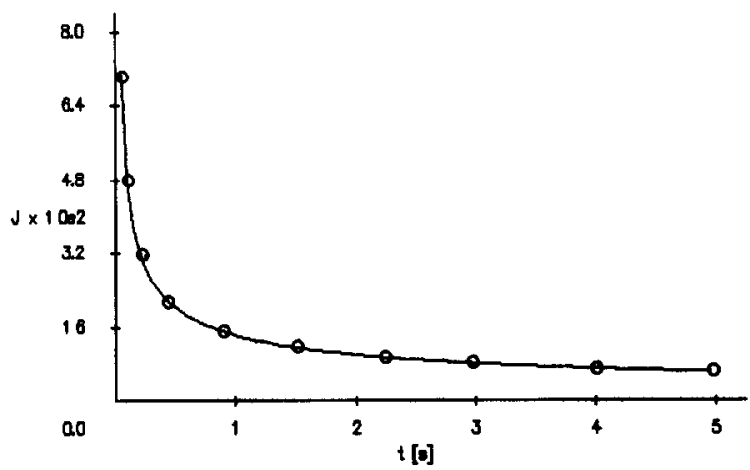

Fig 3 Normalized chronoamperometric curve (-) calculated by numencal solution of Eqn 1 with approximating polynomial of order 4 and $(0)$ results obtained by Cottrell's equation $\left(D=10 \times 10^{-9} \mathrm{~m}^{2} \mathrm{~s}^{-1}\right)$ the characteristic length $(L)$ This fact may cause misunderstandings when chronoamperometric curves of arrays with different characteristic length are compared in figures To avold this problem, the socalled normalized current defined as $J_{t}=I_{t} s_{t} / L$ and which does not depend on $L$ is used in the the present paper for graphical representation of the calculated current-time dependences

The computer program solving numerically Eqn 1 was written in ANSI $\mathrm{C}$ and run on VAX/VMS A program written in Microsoft ${ }^{\circledR}$ QuickC ${ }^{\circledast e r s i o n} 20$ was developed for graph1cal representation of the simulated chronoamperometric results and the concentration distribution of the electroactive species as contour or threedimensional plots Outputs of this program will be presented below

\section{VERIFICATION OF THE MODEL}

As was already mentioned above the current measured by an array of microelectrodes at short times can be calculated by the equation assuming semi-infinite linear diffusion to the electroactive area of the array (Eqn 5) For sufficiently long tımes the current-tıme dependence also obeys Cottrell's equation (Eqn 5) if the whole geometric area of the array is considered as electroactive These theoretical results [30-37], confirmed experimentally by various authors, were used for checkung the validity of the model outlined in the present paper The chronoamperometric curve for an ordered square distribution array of 16 square-shaped microelectrodes with an area of $\mathbf{1 0}$ $\mu \mathrm{m}^{2}$ each was determined by numerical solution of the model The results presented in Fig 4 exactly follow the theoretically and experimentally established behaviour of microarray electrodes This result together with the excellent agreement between the chronoamperometric curves calculated by Cottrell's equation and by numerical solution of the model in the case of a single electrode confirm the validity of the model presented in the present study 


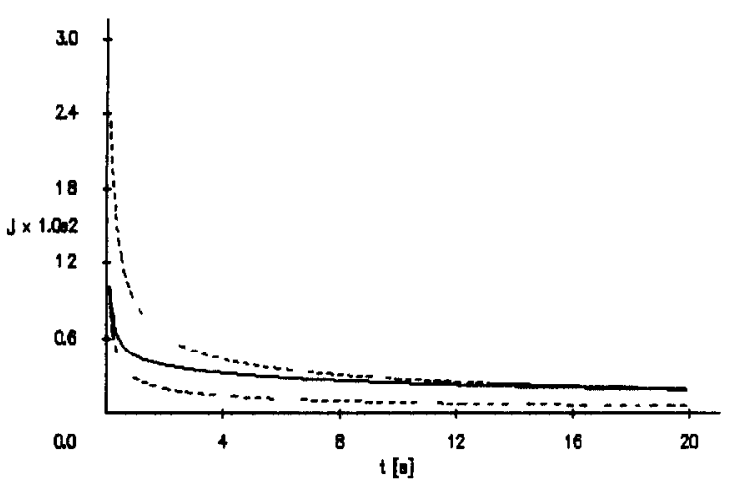

F1g 4 Normalized chronoamperometric curves for a microelectrode array of 16 square distributed square microelectrodes calculated by (- - Cottrell's equation using the electroactive and the total area of the array and (- $\longrightarrow$ ) by the model proposed in this study $\left(D=10 \times 10^{-9} \mathrm{~m}^{2} \mathrm{~s}^{-1}\right)$

\section{ILLUSTRATIVE EXAMPLE}

One of the conditions for proper performance of arrays of amperometric microelectrodes used for multicomponent analysis is munumal interference between the individual microelectrodes This condition will hold if the distance between the electrodes with respect to their size and the diffusion coefficients is big enough. Leaving too big a distance, however, will hamper the miniatunzation of the arrays which is usually aimed at in their construction Thus, the determination of the optimal distance between the electrodes appears to be a key parameter in the designing of the corresponding arrays This can be performed by constructing electrodes with various geometrical dimensions and testing them experimentally $\mathrm{Ob}$ viously this is a costly and time consuming approach The model outlined above is an appropriate tool for the fast and inexpensive solution of this problem For illustrating this fact the chronoamperometric curves of the individual electrodes of two square distributed arrays with equal total electroactive area but with different total area (Fig 5) were calculated For simplicity it was assumed that the electrodes were poised at a potential where the charge-transfer reaction is very fast and the current generation is diffusion controlled The results from the simulations are presented in Fig 6 It can be seen that for array A (Fig 5) there is a clear interference effect resulting in different quasi steady-state currents for the different individual electrodes while for array B (Fig 5) there is an equal accessibility of the electroactive species to all the electrodes resulting in identical chronoamperometric curves From the contour (Fig 5) and the three-dimensional (Fig 7) plots of the concentration field taken at the end of the chronoamperometric numerical experiment at the bottom of the measuring cell, $1 \mathrm{e}, Z=0$, it can be seen that in the case
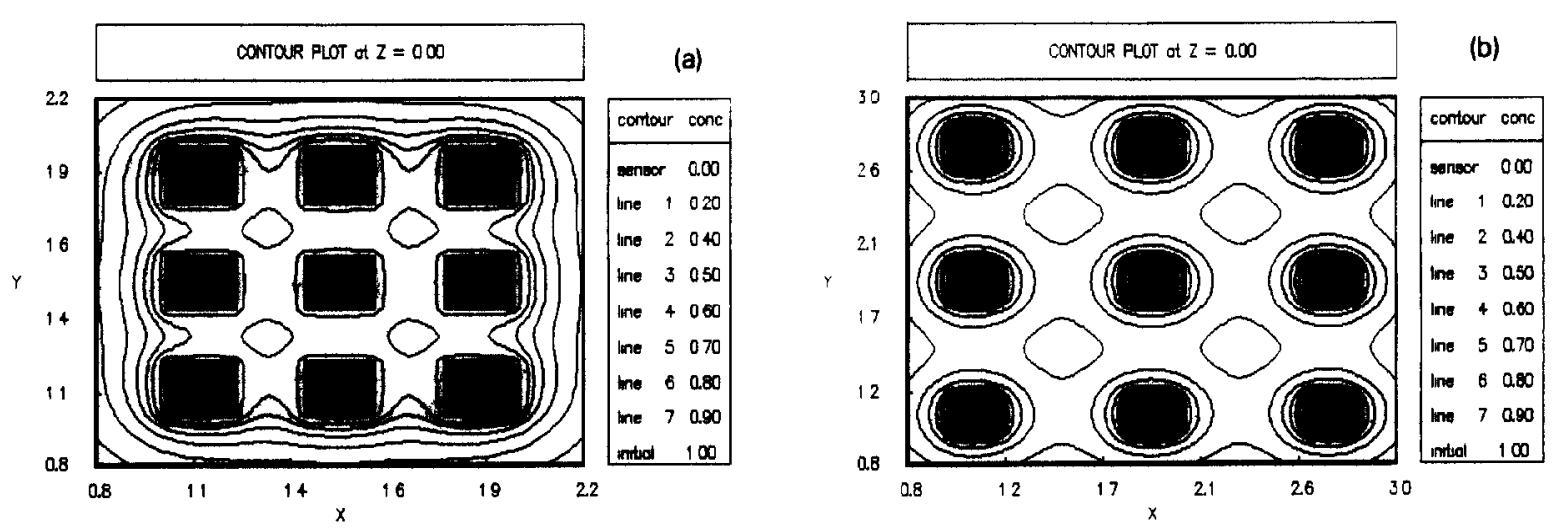

Fig 5 Contour concentration plots at the bottom of the measurng cell at the end of the chronoamperometric numencal experiment $(t=50 \mathrm{~s})$ (a) Array A, (b) array B $\left(D=10 \times 10^{-9} \mathrm{~m}^{2} \mathrm{~s}^{-1}\right)$ (It should be taken into consideration that the microelectrodes of the two arrays are of the same size while the interelectrode gaps differ considerably in size ) 

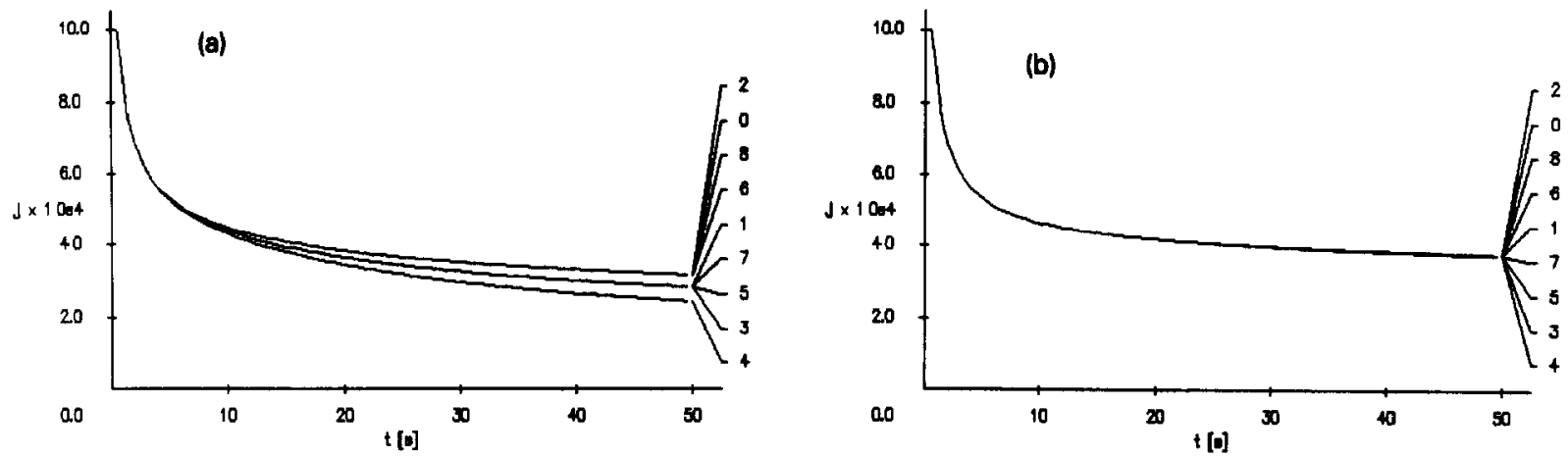

Fig 6 Normalized chronoamperometric curves for the individual electrodes Conditions are the same as in Fig 5

of array A there is very high degree of overlapping of the diffusion fields of the individual microelectrodes For array B only a slight overlapping takes place far from the microelectrodes and it can hardly affect under the conditions of the experiment their behaviour as isolated microelectrodes giving the same amperometric response

\section{Conclustons}

A general mathematical model describing the response of an array of amperometric electrodes with arbitrary distribution placed at the bottom of a measuring cell with rectangular walls and finite dimensions is outlined It consists of the three-dimensional isotropic diffusion equation with boundary conditions corresponding to impermeable walls of the measuring cell and a chargetransfer reaction with either the anodic or the cathodic reaction predominating at the microelectrodes The model allows to take into consideration the influence of the depletion of the electroactive species in the finite volume of the measuring cell on the response of the individual electrodes This feature of the model extends its applicability also to chronocoulometry not treated in the present study Shielding effects due to non-uniform accessibility of the electroactive species to the individual electrodes can be predicted and geometries causing such effects in real arrays can be prevented The simulated chronoamperometric curves show the time required for attaining quasi steady-state current and its value for a given array and measuring cell, thus supplying the necessary information on the duration and sensitivity of analysis

Though in the present study only rectangular
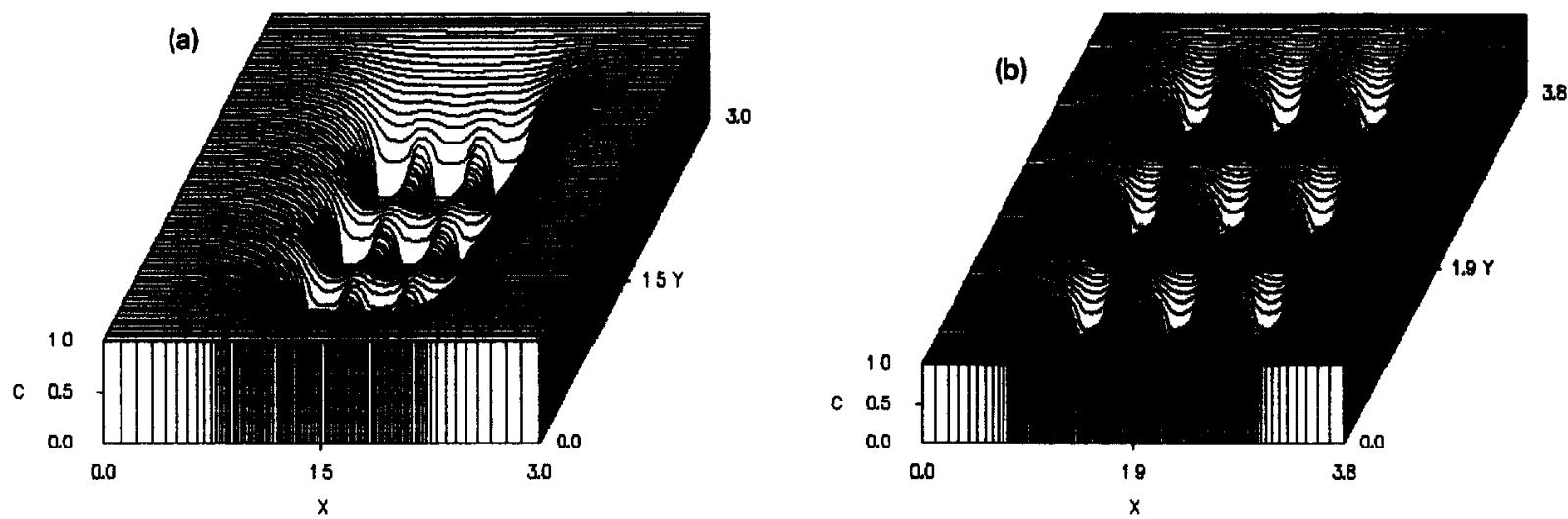

Fig 7 Three-dimensional concentration plots Conditions are the same as in Fig 5 
microelectrodes were considered this fact does not confine the model only to this geometrical shape The response of arrays with microelectrodes of any shape which can be represented as a combination of rectangles can be modelled The model considers only the case of either the anodic or the cathodic reaction predominating which limits its application only to electroanalytical techniques working under such conditions (e g , chronoamperometry, chronocoulometry) but there are no principle obstacles of extending it to reversible and quasi-reversible charge-transfer reactions or to more complex kınetics

\section{REFERENCES}

1 A.G Ewing, MA Dayton and RM Wightman, Anal Chem , 53 (1981) 1942

2 R M Wightman, Anal Chem, 53 (1981) 1125A

3 K.B Oldham, J Electroanal Chem, 122 (1981) 1

4 K. Aok1 and J Osteryoung, J Electroanal Chem, 122 (1981) 19

5 J Heinze, J Electroanal Chem, 122 (1981) 73

6 D Shoup and A. Szabo, J Electroanal Chem, 140 (1982) 237

7 B Speiser and S Pons, Can J Chem, 61 (1983) 156

$8 \mathrm{~K}$. Aokl and J Osteryoung, J Electroanal Chem, 160 (1984) 335

9 H Ikeuchı, M Sato and G P Satô, J Electroanal Chem, 162 (1984) 321

$10 \mathrm{~S}$ Coen, D K. Cope and D E Tallman, J Electroanal Chem , 215 (1986) 29

$11 \mathrm{~K}$. Aok, K. Tokuda and $\mathrm{H}$ Matsuda, J Electroanal Chem, 225 (1987) 19

12 K. Aokı, K. Tokuda and H Matsuda, J Electroanal Chem, 230 (1987) 61

13 J C Myland and K.B Oldham, J Electroanal Chem, 288 (1990) 1

14 J F Cassıdy, S Pons, A.S Hinman and B Speiser, Can J Chem, 62 (1984) 716

$15 \mathrm{~K}$. Aok, K. Akımoto, K. Tokuda and H Matsuda, J Electroanal Chem, 171 (1984) 218

16 M R Deakin, R.M Wightman and C A Amatore, J Electroanal Chem , 215 (1986) 49

17 K. Aokı and K. Tokuda, J Electroanal Chem, 237 (1987) 163
18 P Bindra, A.P Brown, M Fleischmann and D Pletcher, J Electroanal Chem, 58 (1975) 31

19 K.R Wehmeyer, M R Deakın and R M Wightman, Anal Chem , 57 (1985) 1913

20 J Newman, J Electrochem Soc, 113 (1966) 501

21 J O Howell and R M Wightman, Anal Chem, 56 (1984) 524

22 S Bruckenstein, Anal Chem, 59 (1987) 2098

23 A $M$ Bond, $M$ Fleischmann and J Robinson, J Electroanal Chem, 172 (1984) 11

24 C Amatore, M R Deakin and R M Wightman, J Electroanal Chem, 220 (1987) 49

25 N Sleszynska, J Osteryoung and M Carter, Anal Chem, 56 (1984) 130

$26 \mathrm{~W}$ Thormann, $P$ van den Bosch and A M Bond, Anal Chem , 57 (1985) 2764

$27 \mathrm{~T}$ Hepel and J Osteryoung, J Electrochem Soc, 133 (1986) 752

28 R M Penner and Ch R Martın, Anal Chem, 59 (1987) 2625

29 S L Petersen and DE Tallman, Anal Chem, 62 (1990) 459

$30 \mathrm{~J}$ Lindemann and R Landsberg, J Electroanal Chem, 30 (1971) 79

31 E Levart, D Schauhmann, E Contamin and $M$ Etman, J Electroanal Chem, 70 (1976) 117

32 T Gueshı, K. Tokuda and $\mathrm{H}$ Matsuda, J Electroanal Chem , 89 (1978) 247

$33 \mathrm{H}$ Reller, E Kirowa-Eisner and E Gilead, J Electroanal Chem, 138 (1982) 65

34 D E Weisshaar and D E Tallman, Anal Chem, 55 (1983) 1146

35 D Shoup and A. Szabo, J Electroanal Chem, 160 (1984) 19

36 J Cassidy, J Ghoroghchian, F Sarfarazı and S Pons, Can J Chem , 63 (1985) 3577

37 B R Scharifker, J Electroanal Chem, 240 (1988) 61

$38 \mathrm{H}$ Reller, E Kurowa-Eısner and E Gleadı, J Electroanal Chem, 161 (1984) 247

39 J Cassidy, J Ghoroghchian, F Sarfarazı, J J Smith and S Pons, Electrochım Acta, 31 (1986) 629

40 F G Cottrell, Z Physik. Chem, 42 (1903) 385

41 W R Smythe, J Appl Phys, 24 (1953) 70

42 B Carnahan, H.A Luther and JO Wilkes, Applied Numencal Methods, Wiley, New York, 1969

43 S D Kolev and W E van der Lunden, Anal Chem Acta, 247 (1991) 51

44 S D Kolev and WE van der Lunden, Anal Chem Acta, 257 (1992) 331

45 P L T Bran, AIChEJ, 7 (1961) 367

46 A. Savitzky and M J E Golay, Anal Chem, 36 (1964) 1627 